\title{
Impact of a Multimodal Improvement Strategy to Promote Hand Hygiene at a Hospital in Mauritius
}

Dooshanveer C. Nuckchady ${ }^{1}$

1. Infectious Diseases, Victoria Hospital, Quatre-Bornes, MUS

Corresponding author: Dooshanveer C. Nuckchady, doonuckchady@gmail.com

\section{Abstract \\ Introduction}

Limited data is available on which interventions are likely to improve compliance to hand hygiene, especially in underdeveloped countries. The objective of this study is to explore whether the introduction of a particular bundle of strategies to improve hand hygiene is effective.

\section{Material and methods}

In this pre-post study, a multimodal strategy comprised of educating healthcare staff, using reminders, providing feedback and increasing the availability of soap and alcohol, was implemented over a period of one year from 2019 to 2020. Trained observers assessed compliance to hand hygiene before and after the intervention.

\section{Results}

A total of 143 hand hygiene opportunities were observed. Hand hygiene compliance rate did not improve despite the introduction of multiple measures.

\section{Conclusions}

Other approaches should be considered to promote hand hygiene. The choice of which strategies to use should be adapted to the local setting. For instance, in some healthcare facilities, emphasis should be placed on leadership support and on the training of hand hygiene champions.

Categories: Internal Medicine, Infectious Disease

Keywords: hand hygiene, compliance, intervention, multimodal, mauritius

\section{Introduction}

Review began 05/28/2021 Review ended 06/13/2021 Published 06/21/2021

๑) Copyright 2021

Nuckchady. This is an open access article distributed under the terms of the Creative Commons Attribution License CC-BY 4.0., which permits unrestricted use, distribution, and reproduction in any medium, provided the original author and source are credited.
Compliance rate to hand hygiene $(\mathrm{HH})$ in developing countries is known to be poor. This can be a risk factor for the spread of multi-drug resistant organisms. A study conducted at an intensive care unit in Mauritius found a high prevalence of antibiotic resistance: $86 \%$ of Acinetobacter spp., $30 \%$ of Enterobacteriaceae and $80 \%$ of Pseudomonas spp. were carbapenem resistant [1]. Since this is considered a serious issue, a nonrandomized pre-post study was carried out to investigate whether the implementation of multimodal strategies could help to improve HH. Of note, a Cochrane review has suggested that only weak evidence is available to demonstrate the utility of many interventions in ameliorating $\mathrm{HH}$ [2]. Hence, this study adds valuable information to the current literature.

\section{Materials And Methods}

In May 2019, healthcare workers (HCW) at a 600-bed tertiary hospital in Mauritius were observed for one month to assess their compliance to HH. The targeted population included physicians, nurses, and healthcare assistants. All hospital departments including the emergency, intensive care, surgical and internal medicine wards, were involved except for operating theaters, laboratories, and administrative units.

From July 2019 to July 2020, the following steps were introduced: posters on how to perform HH were displayed at each sink, several educational sessions were organized by nursing officers and doctors, alcohol dispensers were made more accessible, feedback regarding the performance on $\mathrm{HH}$ was provided and soap was made available in a larger proportion of toilets. Due to the COVID-19 pandemic, hygiene was emphasized throughout 2020. Training consisted of one-hour PowerPoint presentations; at least 20 participants were invited to attend per session.

In October 2020, for a period of one month, direct observation based on the HH steps described by the World Health Organization (WHO) was once again carried out to determine whether any improvement in $\mathrm{HH}$ occurred. WHO's hand hygiene observation tool was filled in by trained staff [3]. 


\section{Cureus}

A checklist was used to systematically review any barriers that could hinder the implementation of HH. Along with several other items, this checklist assessed the availability of alcohol-based hand rub, the functionality of washbasins, the presence of $\mathrm{HH}$ posters and the accessibility of hand towels for single use.

Data were analyzed using IBM SPSS Statistics version 20 and a p-value of less than 0.05 was considered to be statistically significant.

This study was approved by the Ethics Committee of the Ministry of Health and Wellness of Mauritius.

\section{Results}

Before the intervention, out of $43 \mathrm{HH}$ opportunities, staff used alcohol or washed their hands only 5 times, which constituted a compliance rate of $12 \%$. These opportunities included but were not limited to performing a physical examination, taking vital signs, inserting Foley catheters, placing IV cannulas, and taking glucose readings.

After the intervention, out of $100 \mathrm{HH}$ opportunities, HCW were compliant only once (i.e., $1 \%$ compliance rate; $\mathrm{p}$-value for the $\mathrm{z}$-test to compare proportions using difference $=0.017$ ).

Of note, during that period, the proportion of alcohol dispensers that were functional increased from $64 \%$ to $85 \%$, the number of sinks with a washing agent increased from $34 \%$ to $42 \%$ and the percentage of toilets with soaps increased from $21 \%$ to $29 \%$. Yet, the amount of alcohol used per 1,000 patient days dropped by $18 \%$.

Table 1 summarizes the results of the study and describes the interventions that were instituted.

\begin{tabular}{|c|c|c|c|}
\hline & Pre-intervention & Post-intervention & P-value \\
\hline Number of $\mathrm{HH}^{\star}$ opportunities & 43 & 100 & -- \\
\hline Nursing officers & 13 & 42 & 0.18 \\
\hline Doctors & 22 & 33 & 0.04 \\
\hline Healthcare assistants & 8 & 25 & 0.41 \\
\hline \multicolumn{4}{|l|}{ Iype of interventions } \\
\hline$\%$ of functional alcohol dispensers & $64 \%$ & $85 \%$ & 0.06 \\
\hline$\%$ of beds with alcohol dispensers & $6.3 \%$ & $1.3 \%$ & 0.001 \\
\hline$\%$ of sinks with disposable towel & $0 \%$ & $0 \%$ & 1 \\
\hline$\%$ of sinks with washing agents & $34 \%$ & $42 \%$ & 0.52 \\
\hline$\%$ of toilets with soaps & $21 \%$ & $29 \%$ & 0.56 \\
\hline Training sessions were conducted on $\mathrm{HH}$ & No & Yes & -- \\
\hline Posters were placed on $\mathrm{HH}$ & No & Yes & -- \\
\hline \multicolumn{4}{|l|}{ Outcomes } \\
\hline Amount of alcohol used in $L$ per 1,000 patient-days & 10 & 8.1 & -- \\
\hline HH compliance rate & $12 \%$ & $1 \%$ & 0.017 \\
\hline
\end{tabular}

TABLE 1: This table summarizes the key interventions that were introduced and highlights the outcomes of the study.

${ }^{*} \mathrm{HH}=$ Hand Hygiene.

\section{Discussion}

This is the first study to report the implementation of a multifaceted hand hygiene improvement strategy in the Mauritian healthcare setting. 


\section{Cureus}

HCW were asked to provide an explanation regarding their lack of compliance. Out of the 11 staff that were interviewed, 8 persons (73\%) thought that the lack of functional sanitizers at points of care were one of the main reasons for the failure to adhere to $\mathrm{HH}$ and 3 persons (27\%) believed there was insufficient institutional emphasis, inadequate training and a lack of guidelines on the topic.

During the period of intervention, despite constant advocacy to provide adequate resources to improve $\mathrm{HH}$, the number of alcohol dispensers within 1 meter of a patient bed dropped by 5 times, mostly because the dispensers were preferentially hoarded next to the nursing station. Moreover, disposable paper towel remained unavailable at the washing basins and in toilets. Furthermore, attendance during training sessions was often modest, mostly because of a lack of interest on the topic and a general paucity of awareness on the importance of $\mathrm{HH}$.

The negative results of this study can be explained by a lack of accountability of staff, insufficient oversight by the administration over the issue of hygiene, inadequate hospital leadership, a high ratio of patients to nurses and the absence of a full-time, functional Infection Prevention and Control (IPC) team. In addition, due to the mental toll of the COVID-19 pandemic on the HCW and the absence of local cases of SARS-CoV-2 in the community of Mauritius in October 2020, it is possible that staff let their guard down because of alert fatigue. Moreover, the wearing of gloves could have reduced $\mathrm{HH}$ compliance, a behavior that has been reported elsewhere in the literature [4].

Table 2 summarizes the strategies that can be used to improve HH. Implementing a single procedure at a facility is likely to fail; hence, the use of a bundle of techniques should be emphasized. Decision makers at healthcare facilities can pick a set of multimodal strategies from the table based on the specific hurdles being faced at their location. Several institutions have found success in the application of particular tools like the Joint Commission Targeted Solutions tool, WHO's Multimodal Hand Hygiene Improvement Strategy, Continuous Quality Improvement processes and through the use of behavioral change theories like the Behaviour Centered Design and the Health Action Process Approach [5-10].

\section{Interventions to improve hand hygiene}

Training: Education, problem-based or group-based discussions, simulations and coaching using automated gaming technology

Application of behavioral change theory

Auditing, monitoring and feedback, and applying an accurate and transparent quality assurance. Monitoring can be through direct observation, manually through questionnaires or controlled charts, electronically through camera surveillance or via an automated technique through radiofrequency identification technology

Use of posters and other forms of reminders

Competition e.g., through photo contests or regular display of compliance data

Strong leadership, shared accountability, frontline ownership and frequent goal setting

Broad vertical and horizontal communication with provision of a safe hospital environment

Increasing the availability of hand hygiene supplies, placing dispensers at points of care and proper surveillance of the quantity of products in store

Robust administrative and organizational support

Promotional campaigns

Financial incentives

Coaching handwashing champions and role models

Implementing an institutional operational plan to monitor progress e.g., through the use of a standardized register form of hand hygiene corrective actions

TABLE 2: List of interventions that may be used to improve compliance to hand hygiene in the healthcare setting

This is not the first study to demonstrate a drop in IPC standards during the COVID-19 pandemic. Hess et al. reported a decrease in the use of $\mathrm{HH}$ monitoring badges from 2019 to 2020 while Patel et al. described an increase in the standardized infection ratio of central line-associated bloodstream infections by $28 \%$ when comparing data of 2020 to those of 2019 [11,12]. In France, the HH rate dropped by 9.87\% post-lockdown 
after the first wave of COVID-19; the authors explained that the behavior of HCW was probably altered based on their perceived risk of catching COVID-19 i.e., compliance to HH correlated with the epidemic curve of SARS-CoV-2 [13]. Moore et al. also noted a fall in the adherence to $\mathrm{HH}$ from $60 \%$ to $54 \%$ as the pandemic of COVID-19 progressed [14].

The spread of multi-drug resistant organisms within certain hospitals in Mauritius can be explained by poor HH. Also, the elevated rate of COVID-19 among HCW (especially during the second wave that started in March 2021) may be linked to deficient IPC standards [15]. Furthermore, additional failings in IPC are likely to play a role in the high prevalence of antibiotic resistance among microbes in the country. For instance, according to an audit in 2020 , only $25 \%$ of patients harboring multi-drug resistant organisms were isolated and the use of barrier precautions was often scanty [16].

One main advantage of this study is the long duration of the study of over a year which aimed at assessing the sustainability of the interventions. In addition to this, it is easily reproducible in most settings. Several studies examined the effects of these strategies over a period of only a few weeks but this fails to account for long term effects. The Hawthorne bias was unlikely to be present given that HCW were unaware when they were being observed. The weaknesses include the fact that this study was carried out at a single center, a control group was absent and its sample size was small. However, the author believes the negative results of this study can help to inform subsequent strategies to improve $\mathrm{HH}$, not only to reduce the prevalence of drug resistant microbes but also to stop the spread of harmful organisms in the community, especially during outbreaks [17].

\section{Conclusions}

In the future, multimodal strategies to tackle deficiencies in $\mathrm{HH}$ should take a different approach from the one taken in this study. For instance, dispensers should be placed at points of care, health behavior change theory should be used to engage staff to help sustain long-term improvement, administrative support must be present and $\mathrm{HH}$ champions should be trained. Other strategies that may improve $\mathrm{HH}$ and require further investigation include the organization of $\mathrm{HH}$ competitions and simulation exercises, and the use of automated electronic hand hygiene compliance monitoring systems. Following this study and through noteworthy support from the WHO, the Ministry of Health and Wellness of Mauritius agreed to set up a National IPC Committee in 2021 in order to further scrutinize IPC practices and to ensure that safe practices are followed judiciously in the country.

\section{Additional Information \\ Disclosures}

Human subjects: Consent was obtained or waived by all participants in this study. Minstry of Health and Wellness of Mauritius issued approval NA. This study was approved by the Ethics Committee of the Ministry of Health and Wellness of Mauritius. Animal subjects: All authors have confirmed that this study did not involve animal subjects or tissue. Conflicts of interest: In compliance with the ICMJE uniform disclosure form, all authors declare the following: Payment/services info: All authors have declared that no financial support was received from any organization for the submitted work. Financial relationships: All authors have declared that they have no financial relationships at present or within the previous three years with any organizations that might have an interest in the submitted work. Other relationships: All authors have declared that there are no other relationships or activities that could appear to have influenced the submitted work.

\section{References}

1. Nuckchady DC, Boolaky SH: The prevalence of multi-drug resistant organisms and their outcomes in an ICU in Mauritius: an observational study. Asian J Med Health. 2020, 21:71-8. 10.9734/ajmah/2020/v18i1130270

2. Gould DJ, Moralejo D, Drey N, Chudleigh JH, Taljaard M: Interventions to improve hand hygiene compliance in patient care. Cochrane Database Syst Rev. 2017, 9:CD005186. 10.1002/14651858.CD005186.pub4

3. World Health Organization. Observation Form. (2009). https://www.who.int/gpsc/5may/Observation_Form.doc.

4. Fuller C, Savage J, Besser S, Hayward A, Cookson B, Cooper B, Stone S: "The dirty hand in the latex glove": a study of hand hygiene compliance when gloves are worn. Infect Control Hosp Epidemiol. 2011, 32:1194-9. $10.1086 / 662619$

5. Anderson R, Rosenberg A, Garg S, et al.: Establishing the Foundation to Support Health System Quality Improvement: using a hand hygiene initiative to define the process. J Patient Saf. 2021, 17:23-9. 10.1097/PTS.0000000000000578

6. Farhoudi F, Sanaei Dashti A, Hoshangi Davani M, Ghalebi N, Sajadi G, Taghizadeh R: Impact of WHO Hand Hygiene Improvement Program implementation: A quasi-experimental trial. Biomed Res Int. 2016, 2016:7026169. 10.1155/2016/7026169

7. Mestre G, Berbel C, Tortajada P, et al.: "The 3/3 strategy": a successful multifaceted hospital wide hand hygiene intervention based on WHO and continuous quality improvement methodology. PLoS One. 2012, $7:$ :e47200. 10.1371/journal.pone.0047200

8. Sands M, Aunger R: Development of a behaviour change intervention using a theory-based approach, Behaviour Centred Design, to increase nurses' hand hygiene compliance in the US hospitals. Implement Sci 


\section{Cureus}

Commun. 2021, 2:23. 10.1186/s43058-021-00124-x

9. von Lengerke T, Lutze B, Krauth C, Lange K, Stahmeyer JT, Chaberny IF: Promoting hand hygiene compliance: PSYGIENE-a cluster-randomized controlled trial of tailored interventions . Dtsch Arztebl Int. 2017, 114:29-36. 10.3238/arztebl.2017.0029

10. Ben Fredj S, Ben Cheikh A, Bhiri S, et al.: Multimodal intervention program to improve hand hygiene compliance: effectiveness and challenges. J Egypt Public Health Assoc. 2020, 95:11. 10.1186/s42506-02000039-W

11. Hess OCR, Armstrong-Novak JD, Doll M, et al.: The impact of coronavirus disease 2019 (COVID-19) on provider use of electronic hand hygiene monitoring technology. Infect Control Hosp Epidemiol. 2020, 1-3. 10.1017/ice.2020.1336

12. Patel PR, Weiner-Lastinger LM, Dudeck MA, et al.: Impact of COVID-19 pandemic on central-lineassociated bloodstream infections during the early months of 2020, National Healthcare Safety Network. Infect Control Hosp Epidemiol. 2021, 1-4. 10.1017/ice.2021.108

13. Huang F, Armando M, Dufau S, Florea O, Brouqui P, Boudjema S: COVID-19 outbreak and healthcare worker behavioural change toward hand hygiene practices. J Hosp Infect. 2021, 111:27-34. 10.1016/j.jhin.2021.03.004

14. Moore LD, Robbins G, Quinn J, Arbogast JW: The impact of COVID-19 pandemic on hand hygiene performance in hospitals. Am J Infect Control. 2021, 49:30-3. 10.1016/j.ajic.2020.08.021

15. COVID- 19: Strengthening Infection Prevention and Control Capacity in Mauritius . (2020). Accessed: March 31, 2021: https://www.afro.who.int/news/covid-19-strengthening-infection-prevention-and-controlcapacity-mauritius.

16. Nuckchady DC: Infection prevention and control: situation analysis at Dr. A. G. Jeetoo Hospital, Mauritius 2019-2020 . 2020. https://health.govmu.org/Documents/Main\%20Page/Communique/HSSP\%20Final\%2004\%20March\%202021.pdf.

17. Bloomfield SF, Aiello AE, Cookson B, O'Boyle C, Larson EL: The effectiveness of hand hygiene procedures in reducing the risks of infections in home and community settings including handwashing and alcohol-based hand sanitizers. Am J Infect Control. 2007, 35:S27-64. 10.1016/j.ajic.2007.07.001 